\title{
Intuitionism and the Modal Logic of Vagueness
}

\section{Susanne Bobzien ${ }^{1}$ (D) $\cdot$ Ian Rumfitt ${ }^{1}$ (D)}

Received: 11 June 2018 / Accepted: 15 March 2019/Published online: 13 February 2020

(C) The Author(s) 2020

\begin{abstract}
Intuitionistic logic provides an elegant solution to the Sorites Paradox. Its acceptance has been hampered by two factors. First, the lack of an accepted semantics for languages containing vague terms has led even philosophers sympathetic to intuitionism to complain that no explanation has been given of why intuitionistic logic is the correct logic for such languages. Second, switching from classical to intuitionistic logic, while it may help with the Sorites, does not appear to offer any advantages when dealing with the so-called paradoxes of higher-order vagueness. We offer a proposal that makes strides on both issues. We argue that the intuitionist's characteristic rejection of any third alethic value alongside true and false is best elaborated by taking the normal modal system S4M to be the sentential logic of the operator 'it is clearly the case that'. S4M opens the way to an account of higher-order vagueness which avoids the paradoxes that have been thought to infect the notion. $\mathbf{S 4 M}$ is one of the modal counterparts of the intuitionistic sentential calculus (IPC) and we use this fact to explain why IPC is the correct sentential logic to use when reasoning with vague statements. We also show that our key results go through in an intuitionistic version of S4M. Finally, we deploy our analysis to reply to Timothy Williamson's objections to intuitionistic treatments of vagueness.
\end{abstract}

Keywords Vagueness $\cdot$ Sorites Paradox $\cdot$ Intuitionistic logic $\cdot$ Modal logic $\cdot$ McKinseyTarski translation $\cdot$ Crispin Wright

Ian Rumfitt

ian.rumfitt@all-souls.ox.ac.uk

Susanne Bobzien

susanne.bobzien@all-souls.ox.ac.uk

1 All Souls College, Oxford OX14AL, UK 
Some philosophers have proposed intuitionistic logic as the one best suited to provide the foundation for a theory of vagueness. ${ }^{1}$ As we remind readers in $\S 1$, that logic provides an elegant solution to the Sorites Paradox which avoids the implausible sharp cut-offs in classically based epistemicist theories. Its acceptance has been hampered by two factors. First, the lack of an accepted semantics for languages containing vague terms has led even philosophers sympathetic to intuitionism to complain that no explanation has been given of why intuitionistic logic is the correct logic for such languages (see $\$ 2$ below). Second, switching from classical to intuitionistic logic, while it may help with the Sorites, does not appear to offer any advantages in dealing with the so-called paradoxes of higher-order vagueness. In this paper we offer a proposal that makes strides on both issues: higher-order vagueness and the question why intuitionistic logic is the correct logic for vague languages. We argue (in §3) that the intuitionist's characteristic rejection of any third alethic value alongside true and false is best elaborated by taking the normal modal system $\mathbf{S 4 M}$ to be the sentential logic of the operator 'it is clearly the case that'. $\mathbf{S 4 M}$ opens the way to an account of higher-order vagueness which avoids the paradoxes that have been thought to infect the notion. S4M is one of the modal counterparts of the intuitionistic sentential calculus (IPC) and we use this fact (in §4) to explain why IPC is the correct sentential logic to use when reasoning with vague statements. In this way, we show that the general principles that inspire an intuitionistic approach to vagueness lead precisely to IPC as the logic of a language containing vague terms, without needing to construct a semantic theory of the standard kind for such a language. In $\S 3$, the sentential logic that underpins $\mathbf{S 4 M}$ is assumed to be classical. This yields the important corollary to our argument, that via S4M a classical logician can come to understand what motivates the claim that intuitionistic logic is the correct logic for reasoning with vague expressions. In $\S 5$, however, we argue that for the proponent of an intuitionistic theory of vagueness, the results of $\$ 4$ demand that the underpinning logic should itself be weakened to intuitionistic logic. We show that the key theorems of $\S 3$ still go through in an intuitionistic version of S4M. Finally, we deploy our analysis to reply to Timothy Williamson's objections to intuitionistic treatments of vagueness in [36].

\section{Wright's Argument for Intuitionism as the Logic of Vague Statements}

In some recent papers, Crispin Wright has drawn attention to a version of the Sorites Paradox that is especially stark and difficult to solve (see [38, 39, 41] and especially [40]). Let $a_{1}, \ldots, a_{100}$ be a sequence of a hundred transparent tubes of paint with the following properties: tube $a_{1}$ is clearly red; tube $a_{100}$ is clearly orange and hence clearly not red; but for each $n$, tube $a_{n+1}$ is only marginally more orange (and hence only marginally less red) than its predecessor $a_{n}$. Indeed, let us suppose that an observer with good eyesight, when viewing any pair of adjacent tubes $a_{n}$ and $a_{n+1}$ together in white light but without comparing them with other tubes, is unable to perceive any difference in colour between them. That is, we suppose that any two adjacent members of the sequence are indiscriminable in colour.

\footnotetext{
${ }^{1}$ The seminal text is [22]. For further elaboration and discussion of Putnam's proposal, apart from the papers by Wright on which we focus below, see [5, 21, 23, 24, 26, 30, 31, 35].
} 
Let $L$ be an interpreted first-order language which contains: the connectives ' $\wedge$ ', ' $\vee$ ', and ' $\neg$ '; the quantifier ' $\exists n$ ' ranging over the natural numbers from 1 to 100 ; numerals standing for each of those numbers; a one-place functional expression ' $\xi+1$ ' with the expected sense; a one-place predicate ' $R \xi$ ' with the sense of ' $\xi$ is red'; and complex singular terms in the form ' $a_{n}$ ' with the sense of 'the $n^{\text {th }}$ tube of paint in the sequence'. In the situation described, the tube $a_{1}$ is clearly red, so we may assert in $L$

(1) $R a_{1}$

The tube $a_{100}$, by contrast, is clearly not red, so we may also assert as a premiss

(2) $\neg R a_{100 \text {. }}$

Let us also consider - not as a premiss, but merely as an assumption, to see where it leads - the formula

$$
\text { (3) } \neg \exists n\left(R a_{n} \wedge \neg R a_{n+1}\right) \text {. }
$$

Let us now consider, also as an assumption,

(4) $R a_{99}$

Applying the rule of $\wedge$-introduction to (4) and (2) yields

(5) $R a_{99} \wedge \neg R a_{100}$.

Applying the rule of $\exists$-introduction to (5) yields

(6) $\exists n\left(R a_{n} \wedge \neg R a_{n+1}\right)$,

which is the contradictory of (3). Since assumption (4) has led to a contradiction), we may discharge (4) and infer

$$
\text { (7) } \neg R a_{99}
$$

with (1), (2), and (3) as the only premisses or undischarged assumptions. By repeating the inferential sub-routine (4)-(7) a further 98 times, we eventually reach ${ }^{2}$

(8) $\neg R a_{1}$.

Again, (1), (2), and (3) are the only premisses or undischarged assumptions in the derivation of (8). (1) and, since assumption (3) has led to a contradiction, we may now discharge it, and infer

\footnotetext{
$\overline{2}$ There have been recent attempts (e.g. $[6,43]$ ) to solve the Sorites Paradox by rejecting the transitivity of entailment. Interesting as these may be, we set them aside in this paper.
} 
(9)

$\neg \neg \exists n\left(R a_{n} \wedge \neg R a_{n+1}\right)$

with (1) and (2) as the only premisses. In classical logic (9) yields

$$
\exists n\left(R a_{n} \wedge \neg R a_{n+1}\right)
$$

\section{by Double Negation Elimination.}

As Wright says, this is an extraordinary result. The only premisses in the derivation just set out are (1) and (2). So, simply from the assertions that the first tube in the sequence is red and that the last tube is not red, we have been able to deduce (10). But (10) says that somewhere in our sequence, a red tube is immediately followed by a nonred tube. The case was set up so that neighbouring tubes are indiscriminable in colour. So (10) implies that some tube is red while a tube indiscriminable in colour from it is not red. To most, this claim seems absurd, or at the least unacceptable, and Wright aptly calls (10) the 'Unpalatable Existential'. Yet we have deduced this unpalatable conclusion by applying the rules of classical logic to the indisputably true premisses (1) and (2). ${ }^{3}$

Up to line (9), all the inferential rules used in the derivation are ones which an intuitionist can accept. Only at the last stage, where Double Negation Elimination is used to move from (9) to (10), is an intuitionistically unacceptable rule applied. This observation takes us to the heart of Wright's proposed solution to the paradox. He supposes that the sequence of tubes is monotonic in the sense that if tube $a_{n}$ is red then $a_{m}$ is also red for any $m<n$, and if tube $a_{n}$ is not red then $a_{m}$ is also not red for any $m>n .{ }^{4}$ For Wright, then, (10) expresses the existence of a 'sharp cut-off' in the extension of the predicate 'red'. We are tempted to deny (10), Wright contends, because we think we know that there is no sharp cut-off between the red members of the sequence and the non-red members. But really, we know no such thing. As the deduction shows, the claim that there is no sharp cut-off contradicts the correct verdicts (1) and (2). On Wright's view, though, we are equally unable to assert that there is any sharp cut-off point between the red and the non-red tubes in our sequence. The correct response, Wright claims, is to refrain both from asserting and from denying 'Some red tube in the sequence is immediately followed by a non-red tube'. That is, we should refrain both from asserting and from denying the Unpalatable Existential (10).

What about formula (3), though? Because it is demonstrably inconsistent with the clearly correct premisses (1) and (2), we should deny (3). Assuming that denying a proposition commits one to asserting its negation, we are then committed to asserting (9). However, in order to reach the genuinely unpalatable (10) we would need to eliminate a double negation; this, Wright argues, we are neither compelled nor entitled to do. Since we cannot assume that statements involving vague expressions are either true or false, 'we should...abstain from

\footnotetext{
${ }^{3}$ Wright's leading example in [40] is a Sorites for 'looks red' rather than 'is red'. We suspend judgement on this kind of example (on which see e.g. [14]). Our interest lies with the highly implausible (10). Note that (10) remains implausible even in a case where a viewer can discern some difference in colour between neighbouring tubes, so long as those differences are too slight to vindicate a claim that a tube is red while its neighbour is not.

${ }^{4}$ For present purposes, we can remain neutral on whether it is reasonable to postulate monotonicity in the case described. Because (10) implies that there is a pair of objects indiscriminable (or sufficiently close, see previous footnote) in colour, one of which is red while the other is not, it is unpalatable even without the assumption of monotonicity.
} 
unrestricted use of the law of double negation elimination' ([40] p.441). Such abstention enables us consistently to deny (3), assert (9), but refrain from asserting or denying (10). This combination of assertions, denials, and abstentions from assertion and denial constitutes, Wright proposes, a coherent response to this 'Paradox of Sharp Boundaries'.

We have here a potentially powerful argument for intuitionistic logic —or, at least, for a logic which resembles it in denying the unrestricted validity of Double Negation Elimination. If it works at all, Wright's argument has wide application. Many ordinary predicates are such that nothing in our ordinary practice with them supports the claim that their extensions have sharp cut-offs. For many of those predicates, it will be possible to construct a Sorites sequence. To any such predicate, Wright's argument will apply: on pain of contradicting clear truths, we must deny that there is no sharp cut-off point, whilst resisting the unsupported assertion that there is such a cut-off. This position is stable only if there are restrictions on the elimination of double negations; so we have an argument in favour of a logic which (like intuitionistic logic) imposes such restrictions.

Although Wright uses the existential quantifier ' $\exists n$ ' in formulating the Paradox of Sharp Boundaries, it ranges over the natural numbers from 1 to 100 inclusive, so all formulae containing it could be replaced by appropriate disjunctions. Thus the 'Unpalatable Existential' would be replaced by the 'Unpalatable Disjunction', $\left(R a_{1} \wedge \neg R a_{2}\right) \vee \ldots \vee\left(R a_{99} \wedge \neg R a_{100}\right)$. Wright's solution to his paradox commits him, then, to the thesis that the intuitionistic sentential calculus, IPC, is the correct sentential logic to use when reasoning with vague statements. A logic for vagueness must eventually cover the quantifiers too, and below we shall say a little about how our analysis could be extended to them. Our focus, however, will be on the thesis that IPC is the correct sentential logic of vagueness.

\section{Dummett's Challenge}

What should we make of this argument for intuitionism? In his reply to [40], Michael Dummett put on record his 'admiration for the beautiful solution of the Sorites advocated by Crispin Wright, clouded by a persistent doubt whether it is correct' ([12] p.453). Let us call a statement vague if a vague expression is used in it. Then what unsettles Dummett is that Wright's suggestion that the logic of vague statements is intuitionistic is not underpinned by any detailed semantic analysis. Wright points to broad analogies between the intuitionists' attitude to mathematical statements and the attitude that he recommends taking towards vague statements: in each case, he says, statements' 'truth and falsity have to be thought of as determined by our very practice, rather than by principles which notionally underlie' that practice ([40] p.441). However, those broad analogies fall well short of a developed semantic theory against which the soundness of a given logic could be assessed. 'It is not enough to show that the Sorites paradox can be evaded by the use of intuitionistic logic; what is needed is a theory of meaning, or at least a semantics, for sentences containing vague expressions that shows why intuitionistic logic is appropriate for them rather than any other logic' ([12] p.453).

We agree with Dummett that it would be nice to have a semantic theory for languages which contain vague expressions. At this point in the philosophical debate

\footnotetext{
${ }^{5}$ Wright's solution is in fact very similar to one Putnam [23] had spelled out in response to the objections in [27] to an earlier proposal in [22].
} 
about vagueness, though, there is no semantic theory that we can appeal to either as empirically well-confirmed or as widely accepted by contributors to the debate. To the contrary, even the outlines of such a theory are a hotly contested matter. There is, then, some interest in seeking another sort of argument for the appropriateness of intuitionistic logic in the present context, one which does not take as a premiss the correctness of any semantic theory for vague expressions.

In this quest, it helps to remember how things stood in debates about the foundations of mathematics in the 1920s and early 1930s. Brouwer, Heyting, and others had started to use intuitionistic logic to avoid what they saw as some unacceptable consequences of classical mathematics. At that stage, though, there was no generally accepted semantics for the language of mathematics. Indeed, there was no generally accepted conception of what a semantic theory for any language should provide. All the same, that did not stop philosophers and logicians from asking why (to adapt Dummett's words) 'intuitionistic logic is appropriate for [mathematical statements] rather than any other logic'.

An interesting answer was suggested by a formal result due to Gödel. In his paper 'An Interpretation of the Intuitionistic Sentential Calculus' [17], he observed that

one can interpret Heyting's sentential calculus by means of the notions of the ordinary [i.e. the classical] sentential calculus and the notion " $p$ is provable" (written $\mathscr{B} p$ ) if one adopts for that notion the following system $\mathfrak{I}$ of axioms:

$$
\begin{aligned}
& 1 \quad \mathscr{B} p \rightarrow p, \\
& 2 \quad \mathscr{B} p \rightarrow . \mathscr{B}(p \rightarrow q) \rightarrow \mathscr{B} q, \\
& 3 \quad \mathscr{B} p \rightarrow \mathscr{B} \mathscr{B} p,
\end{aligned}
$$

[along with the rule of proof: given $\vdash A$, deduce $\vdash \mathscr{B} A$ ] and translates Heyting's primitive notions as follows:

$$
\begin{array}{ll}
\neg p & \sim \mathscr{B} p \\
p \supset q & \mathscr{B} p \rightarrow \mathscr{B} q \\
p \vee q & . \mathscr{B} p \vee \mathscr{B} q \\
p \wedge q & p \cdot q
\end{array}
$$

([17] p.39). ${ }^{6}$ That is to say, one can interpret the intuitionistic sentential calculus IPC in a system of classical logic enriched with an additional unary operator ' $\mathscr{B}$ ' if ' $\mathscr{B}$ ' is understood to mean 'it is provable that' and is assumed to conform to the axioms and rules of the modal propositional logic $\mathbf{S 4}$. Here, 'provable' means 'provable by some correct method', not 'provable in a given formal system $S$ '. As Gödel observes at the end of [17], if a formal system $S$ contains $\mathfrak{I}$, then $\mathscr{B}(\mathscr{B} p \rightarrow p)$ is a theorem of $S$. If $S$ also contains arithmetic, $\mathscr{B}(\mathscr{B}(0 \neq 0) \rightarrow 0 \neq 0)$, i.e. $\mathscr{B} \neg \mathscr{B}(0 \neq 0)$, would be a theorem of $S$. However, if $\mathscr{B}$ means 'is provable in $S$, then by Gödel's own Second Incompleteness Theorem $\mathscr{B} \neg \mathscr{B}(0 \neq 0)$ is a theorem of $S$ only if $S$ is inconsistent.

\footnotetext{
${ }^{6}$ Gödel's use of different symbols for classical and intuitionistic connectives suggests that he held that the negation sign (e.g.) used by a classical logician eo ipso has a different sense from that used by an intuitionist. See [28] Section 7.5 for an argument against this thesis.
} 
Gödel's paper is rather telegraphic; what it shows may be spelled out as follows. Let $t$ be a mapping from the well-formed formulae of IPC to well-formed formulae of the language of $\mathfrak{I}$ which meets the following constraints:

For any sentence letter $p, t(p)=\mathscr{B} p$

$$
\begin{aligned}
& t(A \wedge B)=t(A) \wedge t(B) \\
& t(A \vee B)=\mathscr{B} t(A) \vee \mathscr{B} t(B) \\
& t(A \rightarrow B)=\mathscr{B}(t(A) \rightarrow t(B)) \\
& t(\neg A)=\mathscr{B} \neg t(A) .
\end{aligned}
$$

Gödel demonstrated that whenever IPC $-A, \mathfrak{I} \vdash t(A)$. In modern terms, he showed that if IPC $F A$, then $\mathbf{S 4} \vdash t(A)$, where $\mathbf{S 4}$ is the modal system with ' $\mathscr{B}$ ' serving as the necessity operator. He also conjectured the converse, which was eventually proved by McKinsey and Tarski [19]: IPC $-A$ if S4 $-t(A)$. Rasiowa and Sikorski [25] extended these results to intuitionistic predicate logic, IQC. They first extended Gödel's mapping $t$ to quantified formulae as follows:

$$
\begin{aligned}
& t(\forall x A)=\forall x t(A) \\
& t(\exists x A)=\exists x \mathscr{B} t(A) .
\end{aligned}
$$

They then established that IQC $-A$ if and only if QS4 $-t(A)$, where QS4 is got by adding the classical theory of first-order quantification to the classical sentential modal logic S4.

How might these results help to explain why intuitionistic logic is the appropriate system to use when reasoning with mathematical statements? Gödel does not address this question: he presents a logical result and a related conjecture without putting a philosophical gloss on them. All the same, the results just cited suggest the following answer. Whether an individual is entitled to assert a mathematical statement or formula, $A$, will depend on her epistemic state. On one popular view, a speaker is entitled to assert $A$ if, and only if, she knows (or at least knows of) a proof of $A$. We take no stand on the controversial question of what exactly the epistemic requirements are for making a mathematical assertion or for assertions more generally. Whatever one thinks about that, though, it is plausible to hold that a proof of $A$ is the factor to which various speakers must stand in the appropriate epistemic relationship if they are to be warranted in asserting $A$. Let us say, then, that the existence of a proof of $A$ is the extra-mental condition for the assertibility of $A$. On this view, Gödel's translation specifies the extra-mental assertibility conditions for all the well-formed formulae of IPC. Thus the extra-mental condition for an atomic formula to be assertible is that it should be provable (where the conditions for proving various atomic formulae will themselves need to be spelled out); the extra-mental condition for a negated formula to be assertible is that it is provable that its negand is not provable; and so forth.

Let us assume that a formula $A$ counts as logically true when, and only when, the extramental condition for its assertibility is logically guaranteed to obtain. Given this assumption, a classical logician can be brought to understand why an intuitionist does not regard every instance of the Law of Excluded Middle as a logical truth. For an example, let us take the Goldbach Conjecture, which we may formalize as $\forall x \exists y R x y$, where the domain of quantification is the natural numbers and ' $R x y$ ' has the sense of 'if $x$ is an even number greater than 2 , both $y$ and $(x-y)$ are prime numbers'. For any natural numbers $m$ and $n$ there is a simple 
procedure for deciding whether $R m n$, so $R m n$ if and only if it is provable that $R m n$. It follows that $t(\forall x \exists y R x y)=\forall x \exists y \mathscr{B} \mathscr{B} R x y$ which is equivalent to $\forall x \exists y R x y$ itself. Accordingly, $t(\forall x \exists y R x y \vee \neg \forall x \exists y R x y)$ is equivalent to $\mathscr{B} \forall x \exists y R x y \vee \mathscr{\beta} \neg \forall x \exists y R x y$. By hypothesis, $t(A)$ specifies the extra-mental condition for the assertibility of $A$. In the present case, this condition obtains only if the Goldbach Conjecture is either provable or refutable. Even for the classical logician, however, there is no logical guarantee that the Conjecture is either provable or refutable. In this way, the classical logician can come to understand why the intuitionist does not regard the present instance of Excluded Middle as a logical truth.

If this sort of argument is to help explain deviations from classical logic, then the translation $t$ must provide a plausible account of the extra-mental conditions for asserting the relevant sentences or formulae, as they are used by the relevant speakers. We do not ourselves believe that translation $t$ is a particularly faithful rendering of these conditions: for one thing, it omits the important point that an intuitionist is prepared to assert an existentially quantified formula only when she has a method for constructing a verifying instance. That is one reason why Gödel's interpretation of the language of intuitionistic mathematics has been superseded by semantic theories which capture the intended meanings of the connectives and quantifiers more accurately. All the same, the failure of one application of this method of interpreting a language modally does not mean that others might not succeed. One goal of this paper is to propose a non-mathematical application of the method.

In considering whether a Gödel-type modal translation might explain why intuitionistic logic is the right logic to use when reasoning with vague predicates, it helps to bear two formal facts in mind. First, Gödel's own scheme of translation is not the only scheme that yields comparable results. In fact, McKinsey and Tarski used an alternative mapping, $\tau$ below, from the language of IPC into the language of S4 (whose necessity operator we will henceforth write as ' $\square$ '):

(i) For any sentence letter $p, \tau(p)=\square p$

(ii) $\tau(A \wedge B)=\tau(A) \wedge \tau(B)$

(iii) $\tau(A \vee B)=\tau(A) \vee \tau(B)$

(iv) $\tau(A \rightarrow B)=\square(\tau(A) \rightarrow \tau(B))$

(v) $\tau(\neg A)=\square \neg \tau(A)$

It is easy to show that S4 $-\tau(A) \leftrightarrow \square t(\mathrm{~A})$, so we also have that IPC $P A$ if and only if S4 $-\tau(A)$. Because it is simpler, the McKinsey-Tarski mapping has largely superseded Gödel's original, and $\tau$ will prove to be a more convenient translation for our purposes than $t$. Note that $A_{1}, \ldots, A_{n} \vdash_{\text {IPC }} B$ if and only if $F_{\text {IPC }}\left(A_{1} \wedge \ldots \wedge A_{n}\right) \rightarrow B$, and $F_{\mathrm{S} 4} \tau\left(\left(A_{1} \wedge \ldots \wedge A_{n}\right) \rightarrow B\right)$ if and only if $F_{\mathbf{S} 4} \square\left(\tau\left(A_{1}\right) \wedge \ldots \wedge \tau\left(A_{n}\right) \rightarrow \tau(B)\right)$ if and only if $F_{\mathbf{S} 4} \tau\left(A_{1}\right) \wedge \ldots \wedge \tau\left(A_{n}\right)$ $\rightarrow \tau(B)$ if and only if $\tau\left(A_{1}\right), \ldots, \tau\left(A_{n}\right) \vdash_{\mathbf{S} 4} \tau(B)$. So $A_{1}, \ldots, A_{n} \vdash_{\mathbf{I P C}} B$ if and only if $\tau\left(A_{1}\right), \ldots, \tau\left(A_{n}\right) F_{\mathrm{S} 4} \tau(B)$.

Second, and more importantly, S4 is not the only modal logic which corresponds to intuitionistic logic in the specified way. Let us call a modal sentential logic ML a modal companion of intuitionistic logic whenever IPC $-A$ if and only if ML $-\tau(A)$. Then intuitionistic logic has an infinitude of modal companions. Let Grz be the Grzegorczyk Axiom: 


$$
\square(\square(A \rightarrow \square A) \rightarrow A) \rightarrow A .
$$

Then any modal logic in the closed interval whose weakest member is $\mathbf{S 4}$ and whose strongest member is $\mathbf{S 4}+\mathbf{G r z}$ is a modal companion of IPC. ${ }^{7}$ There are similar results for IQC and indeed for the entire class of 'superintuitionistic' logics, i.e. those which are intermediate in strength between the intuitionistic and classical systems. In applying Gödel's method to try to make sense of why intuitionistic logic might be the right one to use in a given context, one is not confined to $\mathbf{S 4}$. Any modal logic between $\mathbf{S 4}$ and $\mathbf{S 4}+$ Grz will serve. Since any such logic includes the rule of necessitation, we shall again have, for any modal companion ML of IPC, the result that $A_{1}, \ldots, A_{n} t_{\text {IPC }} B$ if and only if $\tau\left(A_{1}\right), \ldots, \tau\left(A_{n}\right) \vdash_{\mathrm{ML}} \tau(B)$.

In the results just cited, the underlying logic of the various modal companions is taken to be classical. In pursuing our subsidiary project of explaining to a classical logician why Wright's philosophical assumtions lead precisely to IPC as the appropriate non-modal sentential logic for a language with vague terms, we shall adopt this assumption until the end of $\S 4$. In $\S 5$, however, we shall ask what might be the appropriate companion extension of IPC, in which the intuitionistic sentential logic is taken to underlie the modal system.

\section{The Modal Logic of Vagueness}

How might $\tau$ vindicate the claim that IPC is the correct logic to use when reasoning with vague terms? In outline, our argument will run as follows. Let us interpret the symbol ' $\square$ ' of the modal language as meaning 'it is clear that' or 'it is definite that' (terms which we use interchangeably). We first contend that, on Wright's philosophical assumptions, and where $A$ is a sentence or formula that contains a vague term, $\tau(A)$ gives the extra-mental condition for $A$ to be assertible. Thus, applying this principle to the language $L$ of $\S 1$, the extra-mental condition for ' $R a_{50}$ ' to be assertible (as a formula of $L$ ) will be that it is clear that tube $a_{50}$ is red; the corresponding condition for ' $\neg R a_{50}$ ' is that it is clear that it is not clear that tube $a_{50}$ is red; and so forth. As in the mathematical case, whether a particular language user is entitled to assert such a statement will depend on her epistemic situation: a blind speaker may not be entitled to assert ' $R a_{1}$ ' even though tube $a_{1}$ is clearly red. All the same, we hypothesize, $\tau(A)$ spells out the factor to which speakers must stand in the appropriate epistemic relationship if they are to be warranted in asserting $A$. Support for this hypothesis, which we will lay out in $\S 4$, comes from the sense it makes of the assertive behaviour of competent users of $L$. Second, we argue that the correct modal logic of the operator 'it is clear that' is one of the modal companions of IPC. Putting these things together, we conclude that IPC is the correct logic for a language such as $L$. In this way, we answer Dummett's challenge to 'show why intuitionistic logic is appropriate for [vague terms] rather than any other logic'. The argument rests on philosophical premisses which adherents of classical logic will resist. Given those premisses, though, it vindicates the precise choice of IPC as the appropriate logic to use when reasoning with vague predicates.

\footnotetext{
${ }^{7}$ See Theorem 9.61 and Corollary 9.64 of [4]. Chagrov and Zakharyaschev give their results for what they call 'the Gödel interpretation' of the language of IPC, but in fact the translation they use is McKinsey and Tarski's: see [4] p.96.
} 
It is natural to use the notion of clarity in spelling out the extra-mental conditions for the assertibility of vague statements. However, a distinctive feature of our approach is that clarity — and definiteness, and determinacy — are to be explained in terms of being a borderline case of a property. We take this last notion to be philosophically basic. There are distinctive marks of borderlineness: competent speakers hedge, or otherwise manifest uncertainty, when called upon to apply a predicate that signifies a given property to one of its borderline cases. They might allow that different speakers may reasonably have different views about whether the predicate is correctly applicable to such a case. We express no view on the reasons for this hedging behaviour. Rather, we take the hedging per se to be constitutive of something's being borderline.

In constructing the modal language in which we spell out the extra-mental conditions for assertibility of vague sentences, we take as primitive a sentence-forming operator ' $\nabla$ ' with the sense of 'it is borderline whether'. (We choose the symbol ' $\nabla$ ', pronounced 'nabla', because 'it is borderline whether' stands to 'it is clearly the case that' as 'it is contingent whether' stands to 'it is necessarily the case that'. ${ }^{8}$ ) What axioms and rules govern $\nabla$ ? First, it is highly plausible to maintain that, where $A$ is a theorem of a satisfactory logic of borderlineness, the truth of $A$ is not a borderline matter; we expect truths of logic to be determinately true. We thus have the following rule of proof:

$$
\nabla \mathbf{R} \quad \text { Given } \vdash A \text {, infer } \vdash \neg \nabla A \text {. }
$$

Second, we understand the notion of its being borderline whether such-and-such is the case so as to be neutral between affirmative and negative verdicts. Thus we have the schema.

$$
\nabla 1 \quad \nabla A \leftrightarrow \nabla \neg A .
$$

Third, we postulate.

$$
\text { ק2 } \quad A \rightarrow(\neg \nabla(A \rightarrow B) \rightarrow(\neg \nabla A \rightarrow \neg \nabla B)) .
$$

The justification for schema $\mathbf{\nabla 2}$ is as follows. Suppose it is not borderline whether $A \rightarrow B$. Suppose further that $A$. Then the determinate status of $A \rightarrow B$ cannot be down to $A$ 's being determinately false. Indeed, given $\neg \nabla A, A$ must be determinately true. But if $A$ is determinately true, and $A \rightarrow B$ is determinately true or determinately false, then $B$ must either be determinately true or determinately false. So it is not borderline whether $B$. The basic logic of borderlineness is completed by the schema.

$$
\nabla 3 \quad \nabla(A \vee B) \rightarrow(\nabla A \vee \nabla B) .
$$

Again, this principle is compelling. If neither $A$ nor $B$ were borderline, then $A \vee B$ would either be determinately true (if one or other of $A$ and $B$ were determinately true) or determinately false (if both $A$ and $B$ were determinately false).

It would be possible to develop our theory with ' $\nabla$ ' as the sole modal operator. That approach, however, would have the considerable demerit of making it hard to compare our theory with other work in this area. Comparison is aided by defining a sentential operator, ' $\square$ ', with the intended sense of 'it is clear that', as follows: ' $\square A$ ' means ' $A \wedge \neg \nabla A$ '. We may also define ' $\nabla A$ ' to mean ' $A \vee \nabla A$ '. Given $\nabla \mathbf{1}$ and assuming classical sentential logic, ' $\square$ ' and ' $\nabla$ ' are duals: we have both $\neg \square A \leftrightarrow \diamond \neg A$ and $\square \neg A \leftrightarrow \neg \diamond A$.

\footnotetext{
$\overline{{ }^{8} \text { Useful essays on contingency logic are }[20,44] .}$
} 
An immediate consequence of these definitions is that $\nabla A \wedge \neg \square A$ is equivalent to $\nabla A$. To establish the equivalence from right to left, suppose $\nabla A$. Since $\nabla A$ is defined as $A \vee \nabla A, \diamond A$ follows by $\vee$-introduction. Since $\square A$ is defined as $A \wedge \neg \nabla A, \square A$ implies $\neg \nabla A$ by $\vee$-elimination, whence $\neg \neg \nabla A$ implies $\neg \square A$. Given $\nabla A$, then, double-negation introduction yields $\neg \neg \nabla A$ and hence $\neg \square A$. Finally, by $\wedge$-introduction, $\nabla A$ implies $\nabla A \wedge \neg \square A$. (Note that the proof of this half of the equivalence is acceptable to an intuitionist.) For the converse result, suppose $\diamond A \wedge \neg \square A$. By $\wedge$-elimination, this yields $\nabla A$, i.e. $A \vee \nabla A$. $A \vee \nabla A$ and $\neg \nabla A$ together entail $A$, and hence $A \wedge \neg \nabla A$. That is, $\diamond A$ and $\neg \nabla A$ together entail $\square A$. By contraposition this shows that $\nabla A$ and $\neg \square A$ together entail $\neg \neg \nabla A$, so that $\diamond A \wedge \neg \square A$ entails $\nabla A$, as required. ${ }^{9}$

The definition of $\square$, along with our postulates for $\nabla$, also yield some familiar modal principles. Conjunction elimination yields schema $\mathbf{T}$ :

$$
\mathbf{T} \square A \rightarrow A .
$$

Conjunction introduction combines with $\nabla \mathbf{R}$ to yield the rule of necessitation:

$$
\text { N Given } F A \text {, infer } \vdash \square A \text {. }
$$

It is easy to verify that $\mathbf{\nabla 2}$ yields schema $\mathbf{K}$ :

$$
\text { K } \square(A \rightarrow B) \rightarrow(\square A \rightarrow \square B) .
$$

But what about other principles involving ' $\square$ '?

The modal axiom 4 says that

$$
4 \square A \rightarrow \square \square A .
$$

Should we also assume that the notion of clarity conforms to 4 ? Otherwise put, if it is clearly the case that $P$, is it clearly the case that it is clearly the case that $P ?^{10}$ Given our definition of ' $\square$ ', the question reduces to the correctnesss of the following schema ${ }^{11}$ :

\footnotetext{
9 The last step of this argument assumes a classical logic, but see Section 5 below.

${ }^{10}$ Wright is committed to accepting the $\mathbf{4}$ principle for 'it is clear that'. In his analysis of the so-called paradoxes of higher-order vagueness [37], he postulates a rule of proof, DEF, which in our terms may be formulated as follows:
}

DEF Given a derivation of $B$ from $\square A_{1}, \ldots, \square A_{n}$, construct a derivation of $\square B$ from $\square A_{1}, \ldots, \square A_{n}$.

Since there is a derivation of $\square A$ from $\square A$, DEF licenses the construction of a derivation of $\square \square A$ from $\square A$, whence 4 follows by $\rightarrow$-introduction.

DeVidi [8], on the basis of a sketch of a semantic theory, motivates and defends Wright's solution to the Paradox of Sharp Boundaries by relating it to an intuitionistic modal logic. Unlike Wright, however, DeVidi rejects the $\mathbf{4}$ principle for 'it is clear that', so his logic is an intuitionistic version of KT. The resulting theory is consequently very different from that to be developed here.

${ }^{11}$ Sketch of proof. To show that $\nabla \mathbf{4}$ implies $\mathbf{4}$, assume $\mathbf{\nabla 4}$, and suppose $\square A$, i.e. $A \wedge \neg \nabla A$. We need to deduce $\square \square A$, i.e. $\square A \wedge \neg \nabla \square A$. The first conjunct is trivial. For the second, suppose (for a contradiction) that $\nabla \square A$, i.e. $\nabla(A \wedge \neg \nabla A)$. By $\nabla \mathbf{1}$ this yields $\nabla(\neg A \vee \nabla A)$ and hence $\nabla \neg A \vee \nabla \nabla$ A by $\nabla \mathbf{3}$. By $\nabla \mathbf{1}$, the first disjunct implies $\nabla A$, as does the second disjunct, by $\nabla 4$. Given $\square A$, however, we have $\neg \nabla A$. The resulting contradiction establishes $\neg \nabla \square A$, thereby completing the deduction of $\square \square A$.

To show conversely that $\mathbf{4}$ implies $\mathbf{\nabla 4}$, assume $\mathbf{4}$ and suppose that $\nabla \nabla A$. Then, by the equivalence between $\nabla A$ and $\diamond A \wedge \neg \square A$, we have $\diamond(\diamond A \wedge \neg \square A) \wedge \neg \square(\diamond A \wedge \neg \square A)$. By $\wedge$-elimination, this yields $\diamond(\diamond A \wedge \neg \square A)$. Since $\diamond(B \wedge C)$ implies $\diamond B$, we may infer $\diamond \diamond A$, which combines with $\mathbf{4}$ to give $\diamond A$. Since $\diamond(B \wedge C)$ implies $\diamond C$, we may also infer $\diamond \neg \square A$, i.e. $\neg \square \square A$ which, given 4, implies $\neg \square A$. By $\wedge$-introduction, then, we reach $\diamond A \wedge \neg \square A$, which is $\nabla A$, as required. 


\section{$\nabla 4 \quad \nabla \nabla A \rightarrow \nabla A$.}

Otherwise put, if it is borderline whether it is borderline whether $P$, is it borderline whether $P$ ?

The matter is delicate but, since one of us has argued at length for an affirmative answer (see [1]), we here give only a rough sketch of an argument for $\mathbf{\nabla 4}$ as it applies to sentences of the most pertinent form, $F a$. Recall that we interpret $\nabla F a$ as it is borderline whether $\mathrm{Fa}$ ' and take 'borderline whether $\mathrm{Fa}$ ' to be constituted by the range of hedging behaviour of speakers cognitively and perceptually fully competent with regard to $F$ and $a$ (cf $\S 3$ ). Assuming compositionality of $\nabla$, then, $\nabla \nabla F x$ can be paraphrased as ' $x$ is such that speakers competent with $\nabla F$ hedge over applying $\nabla F$ to $x$ ' and $\nabla F x$ can be paraphrased as ' $x$ is such that speakers competent with $F$ hedge over applying $F$ to $x$ '. We maintain that if relevantly competent speakers hedge over whether to hedge when called upon to apply $F$ to $a$, then one expects relevantly competent speakers also to hedge when called upon to apply $F$ to $a$. For, since any relevant cognitive or perceptual incompetence on the side of the speakers has been ruled out, there remain no reasons why, if relevant speakers do not hedge over $F a$, relevant speakers would hedge over whether they hedge over $\mathrm{Fa}$.

The modal logic of clarity is, then, at least as strong as $\mathbf{S 4} .^{12}$ Is it stronger, though? We shall soon contend that it is, but we first note that any normal modal system which contains every instance of the modal principle $\mathbf{5}$,

$$
5 \quad \diamond A \rightarrow \square \nabla A,
$$

whether as an axiom or as a theorem, is too strong to serve as an adequate logic of clarity. (In particular, then, that logic cannot be the system S5 itself.) This is because, given the definitions of ' $\square$ ' and ' $\nabla$ ', and assuming our postulates for $\nabla, \mathbf{5}$ implies (in fact, it is equivalent to) $\neg \nabla \nabla A$. (For a proof of this equivalence, see [20] Theorem 13.) That is, $\mathbf{5}$ implies that that it is never borderline whether such-and-such is borderline. We do not think that this thesis is tenable. Wright's example of the sequence of tubes of paint shows why. Given the thesis, and assuming that if tube $a_{n}$ is clearly red then $a_{m}$ is also clearly red for any $m<n$, and if tube $a_{n}$ is clearly not red then $a_{m}$ is also clearly not red for any $m>n,{ }^{13}$ the sequence will contain precisely two sharp boundaries, one between the clear cases of red and the clear borderline cases, and the other between the clear borderline cases and the cases which are clearly not red. Since $\mathbf{5}$ precludes us from introducing borderline borderline cases, the sharpness of the transition from clear cases to borderline cases cannot be dulled. Having two such sharp boundaries is no more acceptable than the original single sharp cut-off point between

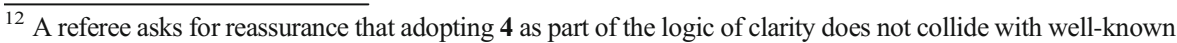
arguments against 'luminosity'. The relationship between borderlineness, luminosity, and 'margin for error' principles is one we have broached elsewhere (in [1] §8). For now, it suffices to remark that we are treating its being clearly the case that $a$ is red simply as the extra-mental condition for asserting ' $a$ is red'. Adopting 4, then, in no way implies that if it is assertible that $a$ is red then it is assertible that it is assertible that $a$ is red, let alone any version of the KK principle.

${ }^{13}$ This assumption - dubbed 'monotonicity of clarity' in [2] -is weaker than Wright's assumption (cf. n.4 above) of monotonicity in respect of redness.
} 
the red and the non-red cases. Therefore any logic with $\mathbf{5}$ as a theorem is too strong to serve as an adequate modal logic of clarity.

Our proposed additional principle says that it is never clearly the case that it borderline whether $P$. In symbols, $\neg \square \nabla A$. We showed earlier that $\nabla A$ is equivalent to $\diamond A \wedge \neg \square A$, so we may reformulate this principle as the schema.

M $\neg \square(\diamond A \wedge \neg \square A)$.

The reader may be surprised at our labelling $\neg \square(\diamond A \wedge \neg \square A)$ as ' $\mathbf{M}$ '. In modal logic, that letter is usually reserved for the axiom $\square \vee A \rightarrow \diamond \square A)$. Quite so, but $\neg \square(\diamond A \wedge \neg \square A)$ and $\square \vee A \rightarrow \diamond \square A$ are classically equivalent in all normal modal logics. As is well known, $\square(A \wedge B)$ is equivalent in $\mathbf{K}$ to $\square A \wedge \square B$ (for a proof see e.g. [18] p.31). Thus $\square(\diamond A \wedge \neg \square A$ ) is equivalent in $\mathbf{K}$ to $\square \vee A \wedge \square \neg \square A$ and hence $\neg \square(\diamond A \wedge \neg \square A)$ is equivalent in $\mathbf{K}$ to $\neg(\square \vee A \wedge \square \neg \square A)$, which is equivalent in the classical sentential calculus to $\square \diamond A \rightarrow \neg \square \neg \square A$, i.e. $\square \diamond A \rightarrow \diamond \square A$. It will be convenient to take $\neg \square(\diamond A \wedge \neg \square A)$ to be definitive of $\mathbf{M}$. The claim that it is never clearly the case that it is borderline whether $P$ has more obvious implications for the logic of vagueness than the more traditional formulation of $\mathbf{M}$.

Whatever the fate of $\mathbf{M}$ on other assumptions, there is a compelling reason why anyone who adopts an intuitionistic treatment of vagueness should accept it. The intuitionist has just as little time as the classical logician for any alethic value other than true and false. Although she refrains from asserting

Bivalence Every statement is either true or false,

she asserts the principle that Dummett labelled

Tertium Non Datur No statement is neither true nor false. ${ }^{14}$

(The inference from Tertium Non Datur to Bivalence is invalid in intuitionistic logic.) In other words, the intuitionist rejects any third alethic value, such as being - alethically - indeterminate, that might stand alongside truth and falsity. Wright is commendably clear about this. Any third status, he writes, is 'un premier pas fatal. It is quite unsatisfactory in general to represent indeterminacy as any kind of determinate truthstatus - any kind of middle situation, contrasting with both the poles (truth and falsity) - since one cannot thereby do justice to the absolutely basic datum that in general borderline cases come across as hard cases: as cases where we are baffled to choose between conflicting verdicts about which polar verdict applies, rather than as cases which we recognize as enjoying a status inconsistent with both' ([38] p.70).

Suppose, then, that there were even a single example, $A$, of a statement which is clearly, or determinately, borderline. Then, because the borderline character of $A$ is determinate, there would be a third alethic value to set alongside true and false. That would controvert Tertium Non Datur and bring to ruin the intuitionistic approach to the topic. Since this would follow even from the

\footnotetext{
${ }^{14}$ Dummett distinguished this semantic principle from the logical law that it is not the case that neither $A$ nor not $A$, for which he proposed the title 'Law of Excluded Third' ([10] p. xix). This proposal never caught on.
} 
existence of a single such statement, a modal logic of clarity that is true to the approach must include a principle which rules out all clearly borderline statements. This is what $\mathbf{M}$ does.

M implies that being borderline exhibits a particular kind of logical elusiveness. As we saw, $\mathbf{M}$ may be written as $\neg \square \nabla A$. Now suppose $\nabla A$. Given the classical duality of ' $\square$ ' and ' $\nabla$ ', the $\mathbf{T}$ axiom entails $B \rightarrow \diamond B$ (for a proof see e.g. [18] p.42). Thus our supposition yields $\diamond \nabla A$. Hence $\mathbf{M}$ and $\mathbf{T}$ together with the supposition $\nabla A$ classically entail $\diamond \nabla A \wedge \neg \square \nabla A$, i.e. $\nabla \nabla A$. Thus $\mathbf{M}$ entails $\nabla A \rightarrow \nabla \nabla A$ in $\mathbf{T}$. Conversely, let us take $\nabla A \rightarrow \nabla \nabla A$ as a premiss and suppose $\square \nabla A$. In $\mathbf{T}, \square \nabla A$ entails $\nabla A$, whence $\nabla \nabla A$, given the premiss. $\nabla \nabla A$ is by definition $\diamond \nabla A \wedge \neg \square \nabla A$, which yields $\neg \square \nabla A$ by $\wedge$-elimination. Since the supposition $\square \nabla A$ yields $\neg \square \nabla A$, we may infer $\neg \square \nabla A$ outright. $\neg \square \nabla A$, however, is $\mathbf{M}$. Putting these two results together, we deduce that $\mathbf{M}$ is classically equivalent to $\nabla A \rightarrow \nabla \nabla A$ in $\mathbf{T}$. Given $\mathbf{T}$, then, $\mathbf{M}$ says this: if it is borderline whether $P$, then it is borderline whether it is borderline whether $P$. Thus, even when we suppose that it borderline whether $P$, what we suppose cannot straightforwardly be the case. We shall have at best a borderline case of what we are supposing to obtain. $\mathbf{M}$ also entails that if it is borderline whether $P$, it is borderline whether it is borderline (or clear) whether it is borderline (or clear) whether $P$, and so on up. The borderlines with clear cases are in this way blurred.

As the flipside of its rendering borderlines elusive in this sense, $\mathbf{M}$ ensures that we do not have to worry about the perplexities that come with substantial hierarchies of higher-order vagueness, i.e. orders of vagueness that are not logically guaranteed to be co-extensive (see e.g. $\left[15,29,32,33\right.$ and 37]). ${ }^{15}$ The logic confirms that the notions of a borderline borderline case, of a borderline borderline borderline case, etc. are all both intelligible and instantiated for vague predicates, as they should be: we agree with Crispin Wright that the case 'for thinking that higher-order vagueness is per se paradoxical...must, of course, be flawed' ([37] p.139). As already noted (see $n .11$ above), the conditional $\nabla \nabla A \rightarrow \nabla A$ is a theorem of $\mathbf{S 4}$. So in that modal system, $\mathbf{M}$ is equivalent to what we may call the $\nabla \nabla$ Principle ('Double Nabla Principle'), viz. $\nabla A \leftrightarrow \nabla \nabla A$. Assuming that the existence of borderline cases is characteristic of vagueness, the $\nabla \nabla$ Principle implies that 'there is no real hierarchy [of higher-order vagueness]. Nothing gives rise to substantive issues about the level of vagueness appropriate to our familiar examples of vagueness' ([29] p.179).

\section{Consequence for Vague Statements}

With this in place, we return to Wright's thesis that IPC is the correct sentential logic to use when reasoning in a language which contains vague terms. ${ }^{16}$

\footnotetext{
${ }^{15}$ Zardini [42] derives what he takes to be problematical consequences, not from the assumption of clear borderline cases, but from the distinct hypothesis that it is clear that there are borderline cases. His analysis is interesting, but at a number of points he makes inferences that are unacceptable to an intuitionist, so we do not consider it in the present dialectical context.

${ }^{16}$ See again the end of $\S 1$ for why the crucial thesis concerns sentential logic.
} 
A logic provides conditions for when a conclusion follows from some premisses. Where $L$ is a sentential language which contains at least one vague expression, and where $A_{1}, \ldots, A_{n}, B$ are formulae of $L$, let us write $A_{1}, \ldots, A_{n} k_{\mathrm{v}} B$ to mean that conclusion $B$ follows from premisses $A_{1}, \ldots, A_{n}$. A logic of vagueness ought to provide conditions for $k_{\mathrm{v}}$. (For present purposes, we need concern ourselves only with the case where a conclusion follows from a finite number of premisses.)

Now if there were an agreed semantic theory for languages containing vague terms, it would be a routine matter to parlay that theory into a necessary and sufficient condition for $k_{\mathrm{v}}$. As remarked in $\S 2$, however, there is no such theory, so we have to proceed less directly.

To see how to proceed, it helps to revert to the explanation touted in $\S 2$ of why Brouwer and others employed intuitionistic logic when reasoning about mathematics. This rested on two postulates: first, that the Gödel translation spells out the extra-mental conditions in which a mathematical sentence or formula $A$ is assertible; second, that a formula $A$ is logically true when, and only when, there is a logical guarantee that the extra-mental condition for its assertibility (the existence of a proof of $A$ ) obtains. This provided the needed explanation, for, when considering the translation, a classical logician will agree that there is no logical guarantee that the extra-mental condition for the assertibility of every instance of Excluded Middle is met. Rather, the logical guarantee extends only to cover the validities of the intuitionistic calculus.

Similarly, we propose, classical logicians can understand why Wright accepts only the rules of intuitionistic logic when reasoning with vague statements, if they adopt the following two postulates. The first connects the translation with the extra-mental condition for the assertibility of vague sentences or formulae: $\tau(A)$ specifies this condition for a vague sentence or formula, $A$, so long as ' $\square$ ' in $\tau(A)$ is read as meaning 'it is clearly the case that'.

Since some of the relevant claims (such as Bivalence and Tertium Non Datur) involve quantifiers, we need to extend $\tau$ so that it covers these operators. We duly propose

(vi) $\tau(\forall x A)=\forall x \tau(A)$

and

(vii) $\tau(\exists x A)=\exists x \tau(A)$.

These clauses stand to the McKinsey-Tarski clauses for ' $\wedge$ ' and ' $\vee$ ' as Rasiowa and Sikorski's treatment of the quantifiers stands to Gödel's original clauses for conjunction and disjunction.

The second postulate says that a sentence or formula in a vague language is logically true when, and only when, there is a logical guarantee that the extra- 
mental condition for its assertibility obtains. More generally, we postulate that an argument in a vague language is logically valid when, and only when, there is a logical guarantee that the extra-mental condition for the assertibility of its conclusion obtains whenever the corresponding conditions for the assertibility of all its premisses obtain. ${ }^{17}$

Why are these two postulates a good way of interpreting Wright? A touchstone for interpreting any intuitionist is to make sense of the fact that she asserts Tertium Non Datur while refraining from asserting Bivalence. When read straight, a classical logician can make no sense of this, because the two statements are classically equivalent, and obviously so. Now, the interpretation we recommend makes this central aspect of the intuitionist's position intelligible, indeed, sensible. When formalized using the obvious notation, and with the domain of quantification restricted to statements, Bivalence is

$$
\forall x(T x \vee F x)
$$

which maps under $\tau$ to

$$
\forall x(\square T x \vee \square F x) .
$$

The classical logician will agree that the condition expressed by the latter formula is not met. The condition in question is that every statement is either clearly true or clearly false. This condition fails if the relevant language contains any vague statements.

Tertium Non Datur, by contrast, is

$$
\neg \exists x \neg(T x \vee F x)
$$

which maps under $\tau$ to

$$
\square \neg \exists x \square \neg(\square T x \vee \square F x),
$$

which is classically equivalent to

$$
\square \forall x \neg \square \neg(\square T x \vee \square F x) .
$$

The condition expressed by this last formula is that it is clearly the case that every statement is not clearly neither clearly true nor clearly false. The condition, in other words, is that it is clearly the case that every statement is not clearly borderline. As explained in $\S 3$, philosophers like Wright, who reject the idea that vague statements possess any third alethic value, hold that this condition is met. Moreover, its being met does not entail that every statement is either clearly true or clearly false, even in classical logic. So the proposed scheme of interpretation

\footnotetext{
${ }^{17}$ If one further assumes that the property which is logically guaranteed to be preserved in a valid argument is truth, then the second postulate implies that a vague statement is true just in case what it says is clearly the case. Not everyone will accept this implication; indeed, rejecting it is one natural way to resist the conclusion that IPC is the correct sentential logic for vague languages. The implication, however, is not so outré as to mar our explanation of why certain philosophical assumptions lead to that conclusion. Dummett ([9], 256) accepts the implication.
} 
provides us with our corollary: it enables the classical logician to understand how Wright can consistently_indeed reasonably-assert Tertium Non Datur, while refraining from asserting Bivalence.

It is vital to this explanation that the scheme of interpretation should map an atomic formula such as ' $T x$ ' to ' $\square T x$ '. So the case provides some confirmation that clause (i) in the specification of $\tau$ is apt for the present purpose. What of the other clauses? Clauses (ii) and (iii) leave the interpretations of conjunction and disjunction unchanged, so no special justification is needed for them. Similarly, (vi) and (vii) leave the interpretations of the universal and existential quantifiers unaffected. However, clause (iv) says that $\lceil A \rightarrow B\urcorner$ maps to $\lceil\square(\tau(A) \rightarrow \tau(B))\rceil$, while clause (v) says that $\lceil\neg A\urcorner$ maps to $\lceil\square \neg A\urcorner$. In the end, the acceptability of any scheme of interpretation has to be assessed holistically. Still, can anything be said to justify prefacing the interpretations of conditional and negated statements with occurrences of 'it is clear that' which do not appear in the formulae that are being interpreted?

We can see why the interpretation scheme for negation is reasonable when we reflect on the Law of Excluded Middle. An intuitionist like Wright is unwilling to assert certain instances of the Law, such as

$$
R a_{50} \vee \neg R a_{50}
$$

with $a_{50}$ supposed to be a borderline case of $R$. If the scheme of interpretation left the sign for negation unchanged, there would be no explanation of this unwillingness, for the condition for asserting ' $R a_{50} \vee \neg R a_{50}$ ' would be:

$$
\square R a_{50} \vee \neg \square R a_{50} .
$$

This formula is another instance of Excluded Middle, so an interpretation that leaves negations unchanged would not help the classicist to understand why intuitionists like Wright are unwilling to assert ' $R a_{50} \vee \neg R a_{50}$ '. Clause (v) of the McKinsey-Tarski translation, however, maps $\lceil\neg A\rceil$ to $\lceil\square \neg A\urcorner$, so the assertibility condition for ' $R a_{50} \vee \neg R a_{50}$ ' will be:

$$
\square R a_{50} \vee \square \neg \square R a_{50}
$$

That is, ' $R a_{50} \vee \neg R a_{50}$ ' will be assertible if and only if either it is clearly the case that tube $a_{50}$ is red or it is clearly the case that it is not clearly red. Since a philosopher who accepts $\mathbf{M}$ will refuse to accept that a borderline case is ever clear, the classical logician can understand why such a philosopher is not prepared to assert this instance of Excluded Middle. ${ }^{18}$

The proposed interpretation of negation also illuminates the way negation interacts with the quantifiers. When the quantification is over a domain of transparent paintfilled tubes, the classicist hears an intuitionist who utters ' $\forall x R x$ ' as saying that every tube is clearly red. When the intuitionist says ' $\exists x R x$ ', the classicist hears her as saying that there is a clearly red tube. Now 'There is a clearly red tube' is clearly incompatible with 'Every tube is clearly not red', which explains why the intuitionist accepts the

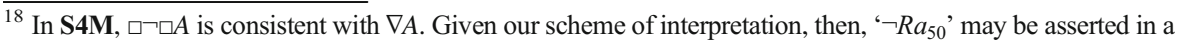
circumstance where it is borderline whether tube $a_{50}$ is red, whereas ' $R a_{50}$ ' may not be. Such an asymmetry may disturb classical logicians, but it is built into any scheme for interpreting intuitionists: the conditions for asserting $\neg A$ cannot simply mirror those for asserting $A$, on pain of there being no explanation of why $\neg A$ is assertible in circumstances where $A$ is not. (We are here indebted to comments from a referee.)
} 
inference from ' $\exists x R x$ ' to ' $\neg \forall x \neg R x$ '. However, 'There is a clearly red tube' does not follow classically from 'Clearly not every tube is clearly not red', which explains why the intuitionist does not accept the converse inference from ' $\neg \forall x \neg R x$ ' to ' $\exists x R x$ '.

What, finally, of clause (iv), which deals with the conditional? This raises issues of a different sort, for few people would maintain that the conditional operator of either classical or intuitionistic logic corresponds at all closely to the vernacular 'if...then'. Both systems validate $\neg(A \rightarrow B) \vDash \neg \neg A$, but we have little inclination to accept the argument 'It is not the case that if the earth is flat, rainbows do not arch; therefore it is not the case that the earth is not flat'. Rather, ' $\rightarrow$ ' is what one might call a logician's conditional, one which validates both modus ponens and a usefully unrestricted rule of conditional proof. Once we appreciate this, we can see that $\lceil A \rightarrow B\rceil$ must map to the modalized formula $\lceil\square(\tau(A) \rightarrow \tau(B))\rceil$ in order for these features to be replicated under the translation. In any event, it is noteworthy that the version of the Sorites Paradox with which we began does not involve a conditional at all. Wright's thesis is that we should use the intuitionistic logic of conjunction, disjunction and negation and in assessing that thesis, the treatment of the conditional is not directly to the point.

There is, then, a strong case for interpreting the intuitionist using the McKinseyTarski translation. Supposing that this interpretation is correct, we can go on to explain (in advance of constructing a semantic theory) why a certain calculus is the correct logic $\mathbf{V}$ for reasoning in $L$. Let $L+$ be the richer language which contains, in addition to all the resources of $L$, the clarity operator ' $\square$ ', and let ML be the modal sentential logic which governs $L+$. Since the conditions for an argument to be valid are spelled out in terms of the extra-mental conditions for the assertibility of the premisses and the conclusion, and since these conditions are in turn given by their translations under $\tau$, we must have

$$
A_{1}, \ldots, A_{n} k_{\mathbf{V}} B \text { if and only if } \tau\left(A_{1}\right), \ldots, \tau\left(A_{n}\right) \vDash_{\mathbf{M L}} \tau(B) \text {. }
$$

In the previous section, we argued that the appropriate modal logic ML is S4M. Hence we reach:

$$
A_{1}, \ldots, A_{n} k_{\mathbf{V}} B \text { if and only if } \tau\left(A_{1}\right), \ldots, \tau\left(A_{n}\right) k_{\mathbf{S} 4 \mathbf{M}} \tau(B)
$$

Now S4M lies in the interval of modal logics between S4 and S4 + Grz. That is, $\mathbf{S 4 M}$ is one of the modal companions of IPC, so that $A_{1}, \ldots, A_{n} \vDash_{\text {IPC }} B$ if and only if $\tau\left(A_{1}\right), \ldots,\left.\tau\left(A_{n}\right)\right|_{\mathbf{S} 4 \mathbf{M}} \tau(B)$. It follows that $\mathbf{V}$ is IPC. In particular, $\mathbf{V}$ cannot be the classical sentential calculus, CPC. If $\mathbf{V}$ were $\mathbf{C P C}$, we should have $F_{\vee} P \vee \neg P$ for any atomic formula $P$. It would then follow that $\tau(P \vee \neg P)=$ $\square P \vee \square \neg \square P$ is a theorem of $\mathbf{S 4 M}$. But $\square P \vee \square \neg \square P$, which is classically equivalent to the $\mathbf{5}$ principle, is not a theorem of $\mathbf{S 4 M}$. (Indeed, we argued that the $\mathbf{5}$ principle is not a theorem of any acceptable modal logic of vagueness.) Hence $\mathbf{V}$ does not validate the Law of Excluded Middle.

What does this argument achieve? By itself, it does not show that Wright is correct to use the intuitionistic sentential calculus, and nothing stronger, when reasoning with vague terms. We contend, though, that it provides an explanation of the kind Dummett sought. His complaint against Wright, it may be recalled, was that 'it is not enough to show that the Sorites paradox can be evaded by the use of intuitionistic logic; what is needed is a theory of meaning, 
or at least a semantics, for sentences containing vague expressions that shows why intuitionistic logic is appropriate for them rather than any other logic' ([12] p.453). What we have done is to identify certain key features of Wright's treatment of vague statements. Although we have not parlayed those features into a standard semantic theory for $L$, we proposed, on their basis, an account of the extra-mental assertibility conditions of the formulae of $L$. By reference to that account, we were then able to show precisely why sentential 'intuitionistic logic...rather than any other <sentential> logic' is appropriate for $L$. The proffered explanation itself does not presume any antecedent commitment to intuitionistic logic. To the contrary, the explanation is one that a classical modal logician could understand and find explanatory. For, throughout the explanation, the sentential logic that underpins $\mathbf{S 4 M}$ is assumed to be the classical propositional calculus, CPC. ${ }^{19}$

\section{An Intuitionistic Modal Logic of Clarity}

This last point raises a question about the coherence of Wright's position. The analysis of $\S 3$ proceeded on the assumption that the sentential logic underpinning the modal logic of clarity would be CPC. Suppose, though, that one takes the plunge and joins Wright in restricting oneself to the weaker system of intuitionistic logic when reasoning with vague statements. Can one then persist in taking the sentential logic underpinning the modal logic to be classical, or must it, too, be weakened to IPC?

There is no formal inconsistency in holding that matters concerning clarity are subject to classical logic, with intuitionistic logic making its appearance only via the McKinsey-Tarski translation into this classical modal language. Indeed, on some philosophical accounts of the nature of clarity, this will be the correct way to go. It seems to us, though, that such a view would not cohere with the philosophical motivations of Wright's position. Remark first that the intuitionist has good reason to introduce notions of clarity, or being borderline, into her language (if they are not already present). As Wright observes, the key to the intuitionistic solution to the Paradox of Sharp Boundaries is that, if she is asked flat out 'Does the sequence contain a tube which is red but whose successor is not red?', the intuitionist can answer neither 'Yes' nor 'No'. Indeed, if she is confined to the simple interpreted language $L$, she is fated to remain silent if asked whether she accepts or rejects formula (10) of $\S 1$. This is, in fact, a pervasive feature of the intuitionistic position. Consider a forced march Sorites - the 'LEM Sorites' - in which an intuitionist is successively asked whether $a_{1}$ is either red or not, whether $a_{2}$ is either red or not,

\footnotetext{
${ }^{19}$ A referee asks whether the theory we put forward is to be understood as normative or descriptive. We are proposing neither that English speakers should nor that most of them do use intuitionistic logic, nor that they should or do use $\mathbf{S 4 M}$, classical or intuitionistic, when reasoning with vague expressions. Rather, given that certain people use intuitionistic logic in such contexts (including rehearsals of Sorites arguments), we advance an explanation of why they do so. It is true that, on our view, the assertibility of borderline cases cannot be established, but neither can their non-assertibility, since it cannot be established whether they are borderline or non-borderline cases. Speakers with a proclivity to assert borderline cases can keep their discourse consistent by adopting a Stalnakerian conversational score policy along the lines suggested by Shapiro in [33].
} 
and finally whether $a_{100}$ is either red or not. The intuitionist will begin by answering 'Yes' (because $a_{1}$ is red) and end by also answering 'Yes' (because $a_{100}$ is not red), but there is a range of intermediate cases in which she has to remain silent. When asked (as it might be) whether $a_{50}$ is either red or not, she cannot answer 'Yes', for an intuitionist may affirm a disjunction only when she is in a position to affirm one or other disjunct. Equally, though, she cannot answer 'No'. Answering 'No' to 'Is it the case that $P$ ?' is tantamount to affirming 'Not $P$ ' but, for the intuitionist as much as for a classicist, the negation of any instance of Excluded Middle yields a contradiction.

There are, then, questions which may be posed in $L$ which an intuitionist cannot answer in $L$. But it is not as though she has nothing to say in response to these questions. She could offer that in such cases she does not assert ' $R a_{50} \vee \neg R a_{50}$ ' because she may be unable to assert either disjunct. She could explain that she may be unable to assert ' $R a_{50}$ ' because it may not be clear that $a_{50}$ is red; and that she may be unable to assert ' $\neg R a_{50}$ ' because it may not be clear that it is not clear that $a_{50}$ is red. Or she may invoke borderlineness for these cases. Such explanations require a language in which the resources of $L$ are reinforced with sentence-forming operators meaning 'it is clear that' and 'it is borderline whether'.

The question then arises what the underlying sentential logic of this richer language is, and it seems to us that on Wright's premisses it ought to be intuitionistic rather than classical. Consider the sentence 'It is clear that tube $a_{30}$ is red', or the corresponding formula ' $\square R a_{30}$ '. Tube $a_{30}$, we may suppose, is a borderline case of clear redness just as tube $a_{50}$ is a borderline case of redness. On the view under consideration, however, we shall be entitled to assert ' $\square R a_{30} \vee \neg \square R a_{30}$ ' and ' $\neg \square R a_{30} \rightarrow \square R a_{30}$ ', even though we may not assert ' $R a_{50} \vee \neg R a_{50}$ ' or ' $\neg R a_{50} \rightarrow R a_{50}$ '. Since, from Wright's perspective, there is no good argument for treating these two kinds of case so differently, we shall explore the hypothesis that the sentential logic underpinning the modal logic of vagueness is IPC, not CPC. We shall then need to check that the key features of Wright's approach to vagueness are retained when the logic underpinning the account of it is weakened in this way.

They are retained. The key feature of the account proposed in $\S 3$ was the equivalence between axiom $\mathbf{M}$ and the $\nabla \nabla$ Principle viz. $\nabla A \leftrightarrow \nabla \nabla A$. We now introduce a plausible intuitionistic modal logic of vagueness and supply derivations that show that this equivalence is retained in it.

In classical modal logic, it is common to select one of the basic modal notions $\square$ and $\diamond$ as primitive and to define the others in its terms. What underpins this are two familiar principles of duality:

$$
\square A \leftrightarrow \neg \diamond \neg A \quad \text { and }
$$

$$
\diamond A \leftrightarrow \neg \square \neg A
$$

The first of these principles, however, undercuts the view we are trying to develop. An intuitionistic consequence of $(\alpha)$ is $\neg \neg \square A \rightarrow \neg \neg \neg \diamond \neg A$. In intuitionistic logic, however, we have $\neg \neg \neg B \leftrightarrow \neg B$ for any formula $B$, so $\neg \neg \neg \checkmark \neg A \rightarrow \neg \diamond \neg A$. Thus $\neg \neg \square A \rightarrow \neg \diamond \neg A$ which yields $\neg \neg \square A \rightarrow \square A$ by ( $\alpha$ ) again (see [3] p.3). Even in the context of intuitionistic logic, then, $(\alpha)$ ensures that Double Negation Elimination may licitly be 


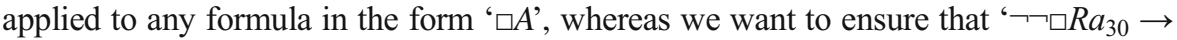
$\square R a_{30}$ ' is not a logical theorem. ${ }^{20}$

In constructing an intuitionistic modal logic, the most common way of getting round this problem is to follow Gisèle Fischer Servi [16] and take both ' $\square$ ' and ' $\Delta$ ' to be primitive operators. In the system we shall present, ' $\square$ ' and ' $\diamond$ ' are not duals. However, neither is primitive. As in the classical case, ' $\square A$ ' is understood to mean $A \wedge \neg \nabla A$ and ' $\nabla A$ ' to mean $A \vee \nabla A$. We now assume, however, that the logic of $\wedge, \vee$, and $\neg$ is intuitionistic, not classical. As we shall see, this enables us to justify a number of the principles which Fischer Servi takes to be axiomatic.

The first point to note is that these definitions ensure that $\nabla A$ remains equivalent to $\diamond A \wedge \neg \square A$ even when the background sentential logic is intuitionistic. An argument given in $\S 3$ (see the text at $n .9$ ) already shows that $\nabla A$ intuitionistically entails $\diamond A \wedge$ $\neg \square A$. As for the converse, we also showed there that $\nabla A \wedge \neg \square A$ intuitionistically entails $\neg \neg \nabla A$. What is more, any formula $\nabla A$ is stable, i.e. we always have $\neg \neg \nabla A \rightarrow \nabla A$. This follows directly from the meaning of ' $\nabla$ ': if it cannot be ruled out that something is not borderline, then it is borderline. Given stability, then, we may complete the argument to establish that $\nabla A \wedge \neg \square A$ intuitionistically entails $\nabla A$. Even in the context of an intuitionistic sentential logic the equivalence between $\nabla A$ and $\nabla A \wedge \neg \square A$ stands, and $\mathbf{M}$ continues to 'say' that there are no clear borderline cases.

Fischer Servi includes the rule of necessitation, $\mathbf{N}$, in her intuitionistic modal system. Given the definition of ' $\square$ ', this is a derived rule of our system: the derivation of $\mathbf{N}$ from $\nabla \mathbf{R}$ is intuitionistically acceptable. She lays down four further principles which, she claims, are needed for a normal intuitionistic modal logic. The first two concern ' $\square$ ':

$$
\begin{aligned}
\mathbf{K} \square \quad \text { (a) } & \square(A \wedge B) \leftrightarrow(\square A \wedge \square B) \\
\text { (b) } & \square \mathrm{T}
\end{aligned}
$$

and the latter two concern ' $\diamond$ '

$\mathbf{K} \diamond$

(a) $\quad \diamond(A \vee B) \leftrightarrow(\diamond A \vee \diamond B)$

(b) From $\diamond \perp$ infer $\perp$.

Given $\mathbf{N}, \mathbf{K} \square(\mathrm{b})$ is redundant. Furthermore, there is an intuitionistically acceptable derivation of $\mathbf{K}$, i.e. $\square(A \rightarrow B) \rightarrow(\square A \rightarrow \square B)$, from $\mathbf{\nabla 2}$, and the standard derivation of $\mathbf{K} \square$ (a) from $\mathbf{K}$ and $\mathbf{N}$ is also intuitionistically acceptable. As for the $\mathbf{K} \diamond$ principles, $\mathbf{K} \diamond(b)$ is clearly acceptable and we can make it a derived rule of our system by laying down the following axiom about borderlineness:

$\nabla 5 \quad \neg \nabla \perp$

This axiom is compelling: since $\perp$ is stipulated to be a falsehood, it is certainly not a borderline matter whether it is true. The left-to-right half of $\mathbf{K} \diamond(\mathrm{a})$ follows directly from $\nabla \mathbf{3}$ and the right-to-left half also follows immediately from the following

\footnotetext{
${ }^{20}$ Note that our definitions of $\square$ and $\diamond$ in terms of $\nabla$ do not have this problematical consequence when the background sentential logic is intuitionistic. While $A \wedge \neg \nabla A$ (i.e. $\square A$ ) intuitionistically implies $\neg(\neg A \vee \nabla \neg A)$ (i.e. $\neg \checkmark \neg A$ ), the converse implication does not hold intuitionistically. That converse, however, is needed to complete the proof that $\neg \square A$ yields $\square A$.
} 
$\mathbf{K} \diamond$-rule $\quad$ Given $\diamond A$ and a derivation of $B$ from $A$, infer $\diamond B$.

This rule of proof is also compelling. Given our definition, ' $\nabla A$ ' may be read as saying ' $A$ cannot be ruled out'. If $A$ cannot be ruled out, and $B$ follows from $A$, then $B$ cannot be ruled out either.

Fischer Servi similarly postulates two principles corresponding to the classical $\mathbf{T}$ axiom:

$\mathbf{T} \square$

$$
\square A \rightarrow A
$$

$\mathbf{T} \diamond$

$$
A \rightarrow \diamond A
$$

These follow immediately from the definitions of ' $\square$ ' and ' $\Delta$ ' in intuitionistically acceptable ways. In the spirit of this paper, we also accept as working hypotheses Fischer Servi's two components of the classical 4 principle:

$$
\begin{array}{ll}
4 \square & \square A \rightarrow \square \square A \\
4 \diamond \quad \triangle \triangleright A \rightarrow \diamond A .
\end{array}
$$

Fischer Servi adds two 'mixing principles' which constrain the interaction of $\square$ and $\diamond:{ }^{21}$

$$
\begin{array}{cc}
\text { Mix1 } & \diamond(A \rightarrow B) \rightarrow(\square A \rightarrow \diamond B) \\
\text { Mix2 } & (\diamond A \rightarrow \square B) \rightarrow \square(A \rightarrow B) .
\end{array}
$$

We are unsure if Mix 2 may be justified given our definitions of $\square$ and $\diamond$ but, since we shall not need it, we leave the matter unresolved. Mix1, however, can be given a justification. It follows intuitionistically from the following principle:

$$
\nabla 6 \quad \nabla(A \rightarrow B) \rightarrow(\square A \rightarrow \nabla B) .
$$

In order to justify $\mathbf{\nabla 6}$, suppose it is borderline whether $A \rightarrow B$, but clear that $A$ is the case. If it were not borderline whether $B$ is the case, then it would either be clear that $B$ is the case or clear that it is not the case. In the first alternative, $A \rightarrow B$ would be clearly the case; in the

\footnotetext{
${ }^{21}$ The best justification we know of Mix 2 is due to Simpson [34]. He formalizes intuitionistic modal logic as a natural deduction system in which the introduction and elimination rules for every connective are 'harmonious' and 'stable' in Dummett's sense. Both Mix1 and Mix2, along with Fischer Servi's other axioms, are theorems of his system. (Mix 1 may be derived using his rules of $\diamond$-elimination, $\square$-elimination, and $\diamond$ introduction; Simpson ([34] p.71) already presents derivations of $\mathbf{K} \diamond(\mathbf{a}), \mathbf{K} \diamond(\mathrm{b})$, and $\mathbf{M i x} \mathbf{2}$ as formulae 4, 3, and 5 respectively.) In [11] ch.13, Dummett had justified IPC precisely on the basis that its natural deduction rules have these properties while those of CPC do not. Simpson's formalization, then, is supported by one influential argument for intuitionistic logic. The Dummettian justification of IPC in terms of harmony, however, is remote from the considerations that have inspired Wright and others to adopt it as the logic of vague statements.

Mix1 and Mix2 are also theorems of Ewald's system of intuitionistic modal logic [13]: apply his definitions of $\square$ and $\diamond$ in terms of tense operators to his tense-logical axioms (G11b) and (G11a) respectively. Ewald, however, advances no philosophical justification for his axioms.
} 
second it would be clearly not the case. We are supposing, however, that it is borderline whether $A \rightarrow B$. The resulting contradiction shows that it is not not borderline whether $B$ is the case. We argued earlier that being borderline is a stable condition. We conclude that it is borderline whether $B$ is the case, which completes the argument for $\nabla \mathbf{6}$.

Mix 1 combines with the $\mathbf{K} \diamond$-rule to yield an important lemma. In IPC, $A$ and $\neg A$ jointly entail $\perp$, so by $\rightarrow$-introduction we have a derivation of $\neg A \rightarrow \perp$ from $A$. By the $\mathbf{K} \diamond$-rule, we may derive $\diamond(\neg A \rightarrow \perp)$ from $\diamond A$. Now an instance of Mix1 is $\diamond(\neg A \rightarrow \perp) \rightarrow(\square \neg A \rightarrow \diamond \perp)$. So $\diamond A$ and $\square \neg A$ together yield $\diamond \perp$, and hence $\perp$ by $\mathbf{K} \diamond$ (b). We have thus proved

\section{Lemma $\quad \diamond A$ and $\square \neg A$ jointly imply $\perp$.}

This lemma is important, for it guarantees that the first conjunct of $\diamond A \wedge \neg \square A$ excludes the possibility that $A$ is clearly false. A key result of $\S 3$ was the equivalence between $\mathbf{M}$ and the thesis $\nabla A \rightarrow \nabla \nabla A$, which says that if something is borderline it is borderline whether it is borderline. In fact, this equivalence is retained even in a weak system of intuitionistic modal logic. Let the system IT be that sentential modal logic whose nonmodal base is IPC and whose modal axioms are $\mathbf{T} \square$ and $\mathbf{T} \diamond$ Then the following are correct derivations in IT:
1. $\nabla A$
Assumption
2. $\nabla A \rightarrow \diamond \nabla A$
Instance of $\mathbf{T} \diamond$
3. $\diamond \nabla A$
(1), (2) modus ponens
4. $\neg \square \nabla A$
M
5. $\diamond \nabla A \wedge \neg \square \nabla A$
(3), (4) ^-introduction
6. $\nabla \nabla A$
(5), equivalence between $\nabla A$ and $\diamond \nabla A \wedge \neg \square A$
7. $\nabla A \rightarrow \nabla \nabla A$
(1), (6) $\rightarrow$-introduction with discharge of assumption (1)
1. $\nabla A \rightarrow \nabla \nabla A$
Premiss
2. $\square \nabla A$
Assumption
3. $\square \nabla A \rightarrow \nabla A$
Instance of $\mathbf{T} \square$
4. $\nabla A$
(2), (3) modus ponens
5. $\nabla \nabla A$
(1), (4) modus ponens
6. $\diamond \nabla A \wedge \neg \square \nabla A$
(5), equivalence between $\nabla A$ and $\diamond A \wedge \neg \square A$
7. $\neg \square \nabla A$
(6) $\wedge$-elimination
8. $\neg \square \nabla A$
(2), (6) reductio ad absurdum, with discharge of assumption (2)

The last line of the second derivation is precisely $\mathbf{M}$, so together the two derivations establish the equivalence of $\mathbf{M}$ and the principle $\nabla A \rightarrow \nabla \nabla A$ in IT. In other words, even in this weak modal logic $\mathbf{M}$ is still tantamount to the principle that if something is borderline, it is borderline whether it is borderline.

What of the converse principle, that if it is borderline whether something is borderline, then it is borderline? In $n .11$ above, we sketched a derivation of $\nabla \nabla A \rightarrow \nabla A$ in classical $\mathbf{S 4}$. We now show that this theorem is also derivable in a system of intuitionistic modal logic which contains both of the 4 principles, $\nabla \mathbf{5}$, the $\mathbf{K} \diamond$-rule, and Mix1. Since $\nabla A$ is equivalent to $\diamond A \wedge \neg \square A$, 
$\nabla \nabla A$ is equivalent to $\diamond(\diamond A \wedge \neg \square A) \wedge \neg \square(\diamond A \wedge \neg \square A)$. We then reason as follows:

1. $\diamond(\diamond A \wedge \neg \square A) \wedge \neg \square(\diamond A \wedge \neg \square A)$ Assumption

2. $\diamond(\diamond A \wedge \neg \square A)$

(1) $\wedge$-elimination

3. $\diamond A \wedge \neg \square A$

Assumption

4. $\diamond A$

(3) $\wedge$-elimination

5. $\diamond \diamond A$

(2), (3), (4) $\mathbf{K} \diamond$-rule with discharge of assumption (3)

6. $\diamond \diamond A \rightarrow \diamond A$

Instance of $\mathbf{4} \diamond$

7. $\diamond A$

8. $\diamond A \wedge \neg \square A$

(5), (6) modus ponens

9. $\neg \square A$

Assumption

10. $\diamond \neg \square A$

(6) $\wedge$-elimination

(2), (8), (9) $\mathbf{K} \diamond$-rule with discharge of assumption (8)

11. $\diamond(\square A \rightarrow \perp)$

(10) IPC, $\mathbf{\diamond} \diamond$-rule

12. $\diamond(\square A \rightarrow \perp) \rightarrow(\square \square A \rightarrow \diamond)$

Instance of Mix 1

13. $\square \square A \rightarrow \diamond_{\perp}$

(11), (12) modus ponens

14. $\square A$

Assumption

15. $\square A \rightarrow \square \square A$

Instance of 4ロ

16. $\square \square A$

(14), (15) modus ponens

17. $\nabla_{\perp}$

(13), (16) modus ponens

18. $\perp$

(17) $\mathbf{K} \diamond(b)$

19. $\neg \square A$

(14), (18) $\neg$-introduction with discharge of assumption (14)

20. $\diamond A \wedge \neg \square A$

(7), (19) $\wedge$-introduction

21. $\diamond(\diamond A \wedge \neg \square A) \wedge \neg \square(\diamond A \wedge \neg \square A) \rightarrow(\diamond A \wedge \neg \square A)$

(1), (20) $\rightarrow$-introduction, with discharge of assumption (1)

This derivation establishes $\nabla \nabla A \rightarrow \nabla A$, as required. Putting the three derivations together, we have shown that in an intuitionistic version of $\mathbf{S 4}$ that is naturally suited to serve as a logic of vagueness, $\mathbf{M}$ is equivalent to the $\nabla \nabla$ Principle, $\nabla A \leftrightarrow \nabla \nabla A$.

In the context of that logic, then, the $\mathbf{M}$ principle still suffices to ensure that 'there is no real hierarchy' of higher-order vagueness (to quote [29] once more). That is, this distinctive implication of $\mathbf{M}$ is retained even as the sentential calculus that underpins the modal logic is weakened from CPC to IPC. ${ }^{22} \mathbf{M}$ also continues to ensure that the borderline cases are elusive in the sense that any attempt to give an example of one will produce, at best, a borderline case of an example.

This last point refutes a recent argument of Timothy Williamson's against an intuitionistic approach to vagueness ([36] pp.32-33). In this argument (one of two),

\footnotetext{
$\overline{{ }^{22}}$ What first-order logic should be built on the foundation of the Fischer Servi-style intuitionistic modal logic S4M? It is suggested (in [2]) that, when the underlying propositional logic is classical, S4M should be supplemented with a finality axiom (for which see [7]), so as to preserve at the first-order level completeness with regard to the same Kripke frames and to extend to the first-order level the desired absence of clear borderline cases. We leave for another day the interesting questions of whether the finality axiom can, or should, play an analogous role in the first-order extension of intuitionistic S4M.
} 
Williamson argues against intuitionistic logic as a basis for denying the Law of Excluded Middle by arguing against intuitionistic logic as the logic we use when reasoning with vague predicates. For him, 'a paradigm borderline case is the worst case for the law of excluded middle' ([36] p.32): all parties, he thinks, will accept that if that law holds for paradigm borderline cases, then it holds for all cases. Let object $a$ be a paradigm borderline case of a vague predicate ' $F$ '. Then, according to Williamson, everyone will accept the conditional

$$
(F a \vee \neg F a) \rightarrow \forall x(F x \vee \neg F x)
$$

Intuitionists, Williamson supposes, will wish to assert that the Law of Excluded Middle 'does not hold always' in cases of vagueness. He takes this to mean that they will wish to assert $\neg \forall x(F x \vee \neg F x)$. However, by $(W)$ and contraposition, this yields $\neg(F a \vee \neg F a)$, the negation of a particular instance of Excluded Middle. As Williamson rightly points out, this is inconsistent in IPC ([36] p.33).

This argument may be resisted at two places (at least). If an intuitionist says that Excluded Middle 'does not always hold' in cases of vagueness, she is not charitably interpreted as meaning to assert $\neg \forall x(F x \vee \neg F x)$. Rather, she is to be understood as saying that we are not entitled to assert arbitrary instances of Excluded Middle when the language contains vague expressions. When so understood, she will not be committed to asserting the negation of the consequent of $(W)$ and there will be no question of applying contraposition to reach a contradictory conclusion. On the view propounded in this paper, however, Williamson's argument does not even get off the ground. The very formulation of $(W)$ assumes that there are paradigm borderline cases for vague terms. In fact, the argument does not require the assumption of a paradigm borderline case; the weaker assumption of any case that is an example of a borderline case of $F$ would do. Either way, intuitionists who favour the modal company of $\mathbf{S 4 M}$ will reject the assumption: no example of a borderline case of $F$ can be given. So, to them Williamson's first argument is irrelevant.

His second argument against intuitionism as a viable basis for a theory of vagueness ([36] p.33) fares no better. It revolves around the question 'Was Mars always either dry or not dry?', in a context where it is assumed that Mars was definitely not dry at some point in the past and has reached its current state of total dryness by a gradual process of evaporation. Williamson's discussion is complicated by the fact that the 'always' in his question is naturally heard as quantifying over a continuum, so we prefer to switch to a simpler case. Imagine a sequence of steps in each of which a single grain of sorghum is removed from what is clearly a heap until only one grain remains, and consider the question 'At every step was there either a heap or not a heap?' Williamson argues that the intuitionist is poorly placed to defend a negative answer: 'the denial of the conjunction of any finite number of instances of the law of excluded middle is intuitionistically inconsistent. The denial of the universal generalization of the law over a finite domain is therefore intuitionistically false too' ([36] p.33).

We agree with Williamson that the intuitionist cannot return a negative answer to this question; she will not, of course, give a positive answer either. But we reject his assumption that a good theory of vagueness has to validate one answer or the other. That assumption simply dismisses intuitionistic theories like Wright's. As we saw in $\S 1$, it is essential to Wright's position that he gives neither of the expected answers to the question 'Is it the case that some red tube in the sequence is immediately followed by a tube which 
is not red?' The negative answer leads to a contradiction, and the positive answer is Wright's 'Unpalatable Existential'. Williamson's question involves a universal, rather than an existential, quantifier but the intuitionist's stance towards it should be the same: she should refrain from answering it negatively, on pain of contradiction, but also refrain from answering it positively, on the ground that we have no reason to believe in the sharp cut-offs that a positive answer implies. That is, the intuitionist neither asserts nor denies that there was either a heap or not a heap at every step. It is true that intuitionistic logic is a poor basis for a negative answer to Williamson's question. Then again, an intuitionist has no reason to give that answer. Williamson's second argument is just as ineffectual as the first against the intuitionist approach to vagueness that has been defended here.

While more work needs to be done, we hope to have shown that that approach is far more promising than Williamson's discussion of intuitionism would suggest. We argued that the general philosophical principles that underpin the intuitionistic approach - in particular, the thesis that borderline cases present no third alethic value to stand alongside true and false - recommend $\mathbf{S 4 M}$ as the correct modal logic of 'it is clearly (definitely, ...) the case that', and does so independently of whether the ambient logic is classical or itself intuitionistic. S4M vindicates the belief that there is higher-order vagueness, while avoiding the hierarchies of higher orders which generate paradox. We were also able to meet Dummett's challenge and explain why IPC is precisely the right logic to use when reasoning with vague terms at the sentential level. Moreover, we were able to do that without constructing a semantic theory for a language with vague terms: the only premiss we needed was that the McKinsey-Tarski translation specifies the conditions in which an intuitionist (such as Wright) who adopts the aforesaid general principles is prepared to assert vague statements. We do not, of course, claim to have shown that intuitionistic logic provides the best solution to the Sorites Paradox: we have not in this paper compared that solution with any of those which retain classical sentential logic. The connection we have drawn between intuitionistic logic and the modal logic of vagueness should make it easier to compare that solution with classical theories which use modal operators to express notions of determinacy, definiteness, or clarity. In any event, the connection already shows that the intuitionistic approach has a logico-philosophical coherence which ought to lead any theorist of vagueness to take it seriously.

Acknowledgements We thank Peter Fritz, Øystein Linnebo, Christopher Peacocke, Sven Rosenkranz, Timothy Williamson, the participants at the workshop Vagueness and Modality: Philosophical Applications of Modal Logic (Oslo, June 2018), and two anonymous referees. We are especially grateful to Crispin Wright, whose talk in Oxford in November 2016 prompted the discussions that resulted in this paper and who offered both encouragement and insightful comments.

Open Access This article is distributed under the terms of the Creative Commons Attribution 4.0 International License (http://creativecommons.org/licenses/by/4.0/), which permits unrestricted use, distribution, and reproduction in any medium, provided you give appropriate credit to the original author(s) and the source, provide a link to the Creative Commons license, and indicate if changes were made. 


\section{References}

1. Bobzien, S. (2012). If it's clear, then it's clear that it's clear, or is it?-higher-order vagueness and the S4 Axiom. In K. Ierodiakonou \& B. Morison (Eds.), Episteme, etc.: Essays in Honour of Jonathan Barnes (pp. 189-212). Oxford: Oxford University Press.

2. Bobzien, S. (Forthcoming 2020). A generic solution to the Sorites paradox based on the normal modal logic Qs4M+BF+FIn. In A. Abasnezhad \& O. Bueno (Eds.), The Sorites Paradox: New Essays, special issue of Synthese (pp. $\mathrm{x}-\mathrm{xx})$. New York: Springer.

3. Bull, R. A. (1965). Some modal calculi based on IC. In J. N. Crossley \& M. A. E. Dummett (Eds.), Formal Systems and Recursive Functions (pp. 3-7). Amsterdam: North Holland.

4. Chagrov, A., \& Zakharyaschev, M. (1997). Modal Logic. Oxford: Oxford University Press.

5. Chambers, T. (1998). On vagueness, sorites, and Putnam's intuitionistic strategy. The Monist, 81, 343-348.

6. Cobreros, P., Egré, P., Ripley, D., \& van Rooij, R. (2012). Tolerant, classical, strict. Journal of Philosophical Logic, 41, 347-385.

7. Cresswell, M. (2001). How to complete some modal predicate logics. In M. Zakharyaschev, K. Segerberg, M. de Rijke, \& H. Wansing (Eds.), Advances in Modal Logic (Vol. 2, pp. 155-178). Stanford: CSLI Publications.

8. DeVidi, D. (2005). Vagueness and intuitionistic logic: on the Wright track. In K. A. Peacock \& A. D. Irvine (Eds.), Mistakes of Reason: Essays in Honour of John Woods (pp. 279-295). Toronto: University of Toronto Press.

9. Dummett, M.A.E. (1975). Wang's Paradox. Synthèse, 30, 301-24. Page references to the reprint in [10], pp.248-68.

10. Dummett, M. A. E. (1978). Truth and Other Enigmas. London: Duckworth.

11. Dummett, M. A. E. (1991). The Logical Basis of Metaphysics. London: Duckworth.

12. Dummett, M. A. E. (2007). Reply to Crispin Wright. In R. E. Auxier \& L. E. Hahn (Eds.), The Library of Living Philosophers: Michael Dummett (pp. 445-454). Chicago: Open Court.

13. Ewald, W. B. (1986). Intuitionistic tense and modal logic. The Journal of Symbolic Logic, 51, 166-179.

14. Fara, D. G. (2001). Phenomenal continua and the Sorites. Mind, 110, 905-935.

15. Fara, D. G. (2001). Gap principles, penumbral consequence, and infinitely higher-order vagueness. In J. C. Beall (Ed.), Liars and Heaps: New Essays on Paradox (pp. 195-221). Oxford: Oxford University Press.

16. Fischer Servi, G. (1977). On modal logic with an intuitionistic base. Studia Logica, 36, 141-149.

17. Gödel, K. (1933). Eine Interpretation des intuitionistischen Aussagenkalküls. Ergebnisse eines mathematischen Kolloquiums, 4, 39-40.

18. Hughes, G. E., \& Cresswell, M. J. (1996). A New Introduction to Modal Logic. London: Routledge.

19. McKinsey, J. C. C., \& Tarski, A. (1948). Some theorems about the sentential calculi of Lewis and Heyting. The Journal of Symbolic Logic, 13, 1-15.

20. Montgomery, H., \& Routley, R. (1966). Contingency and non-contingency bases for normal modal logics. Logique et Analyse, 9, 318-328.

21. Mott, P. (1994). On the intuitionistic solution of the Sorites paradox. Pacific Philosophical Quarterly, 75, $133-150$.

22. Putnam, H. W. (1983). Vagueness and alternative logic. In his Realism and Reason: Philosophical Papers Volume 3 (pp. 271-286). Cambridge: Cambridge University Press.

23. Putnam, H. W. (1985). A quick Read is a wrong Wright. Analysis, 45, 203.

24. Putnam, H. W. (1991). Replies and comments. Erkenntnis, 34, 401-424.

25. Rasiowa, H., \& Sikorski, R. (1953). Algebraic treatment of the notion of satisfiability. Fundamenta Mathematicae, 40, 62-95.

26. Rea, G. (1989). Degrees of truth versus intuitionism. Analysis, 49, 31-32.

27. Read, S. L., \& Wright, C. J. G. (1985). Hairier than Putnam thought. Analysis, 45, 56-58.

28. Rumfitt, I. (2015). The Boundary Stones of Thought: An Essay in the Philosophy of Logic. Oxford: Clarendon Press.

29. Sainsbury, R. M. (1991). Is there higher-order vagueness? The Philosophical Quarterly, 41, 167-182.

30. Schwartz, S. P. (1987). Intuitionism and Sorites. Analysis, 47, 179-183.

31. Schwartz, S. P., \& Throop, W. (1991). Intuitionism and vagueness. Erkenntnis, 34, 347-356.

32. Shapiro, S. (2005). Context, convention, and so-called 'higher-order' vagueness. Proceedings of the Aristotelian Society, Supplementary Volumes, 105, 147-165.

33. Shapiro, S. (2006). Vagueness in Context. Oxford: Clarendon Press.

34. Simpson, A.K. (1994). The Proof Theory and Semantics of Intuitionistic Modal Logic. PhD dissertation, University of Edinburgh. 
35. Williamson, T. (1996). Putnam on the Sorites Paradox. Philosophical Papers, 25, 47-56.

36. Williamson, T. (2007). The Philosophy of Philosophy. Oxford: Blackwell.

37. Wright, C. J. G. (1992). Is higher-order vagueness coherent? Analysis, 52, 129-139.

38. Wright, C. J. G. (2001). On being in a quandary: relativism, vagueness, logical revisionism. Mind, 110, 45-98.

39. Wright, C. J. G. (2003). Rosenkranz on quandary, vagueness and intuitionism. Mind, 112, 465-474.

40. Wright, C. J. G. (2007). Wang's Paradox. In R. E. Auxier \& L. E. Hahn (Eds.), The Library of Living Philosophers: Michael Dummett (pp. 415-444). Chicago: Open Court.

41. Wright, C. J. G. (Forthcoming). Intuitionism and the Sorites. In S. Oms \& E. Zardini (Eds.), The Sorites Paradox. Cambridge: Cambridge University Press.

42. Zardini, E. (2006). Higher-order vagueness and paradox: the glory and misery of S4 definiteness. The Baltic International Yearbook of Cognition, Logic and Communication, 1, 203-220.

43. Zardini, E. (2008). A model of tolerance. Studia Logica, 90, 337-368.

44. Zolin, E. (1999). Completeness and definability in the logic of non-contingency. Notre Dame Journal of Formal Logic, 40, 533-547.

Publisher's Note Springer Nature remains neutral with regard to jurisdictional claims in published maps and institutional affiliations. 